Gnido Amandine Assog BA ${ }^{1}$

Adandé Belarmain FANDOHAN ${ }^{1,2,3}$

Valère Kolawolé SALAKO ${ }^{3}$

Achille Ephrem AsSOGBADJo ${ }^{1,3}$

${ }^{1}$ Université d'Abomey-Calavi Faculté des sciences agronomiques Laboratoire d'écologie appliquée 04 BP 1525, Cotonou

Bénin

${ }^{2}$ Université nationale d'agriculture École de foresterie et ingénierie du bois Unité de recherche en foresterie, agroforesterie et biogéographie BP 45, Kétou

Bénin

${ }^{3}$ Université d'Abomey-Calavi Faculté des sciences agronomiques Laboratoire de biomathématiques et d'estimations forestières 04 BP 1525, Cotonou Bénin

\section{Usages de Bombax costatum (Malvaceae) dans les terroirs riverains de la réserve de biosphère de la Pendjari, République du Bénin}

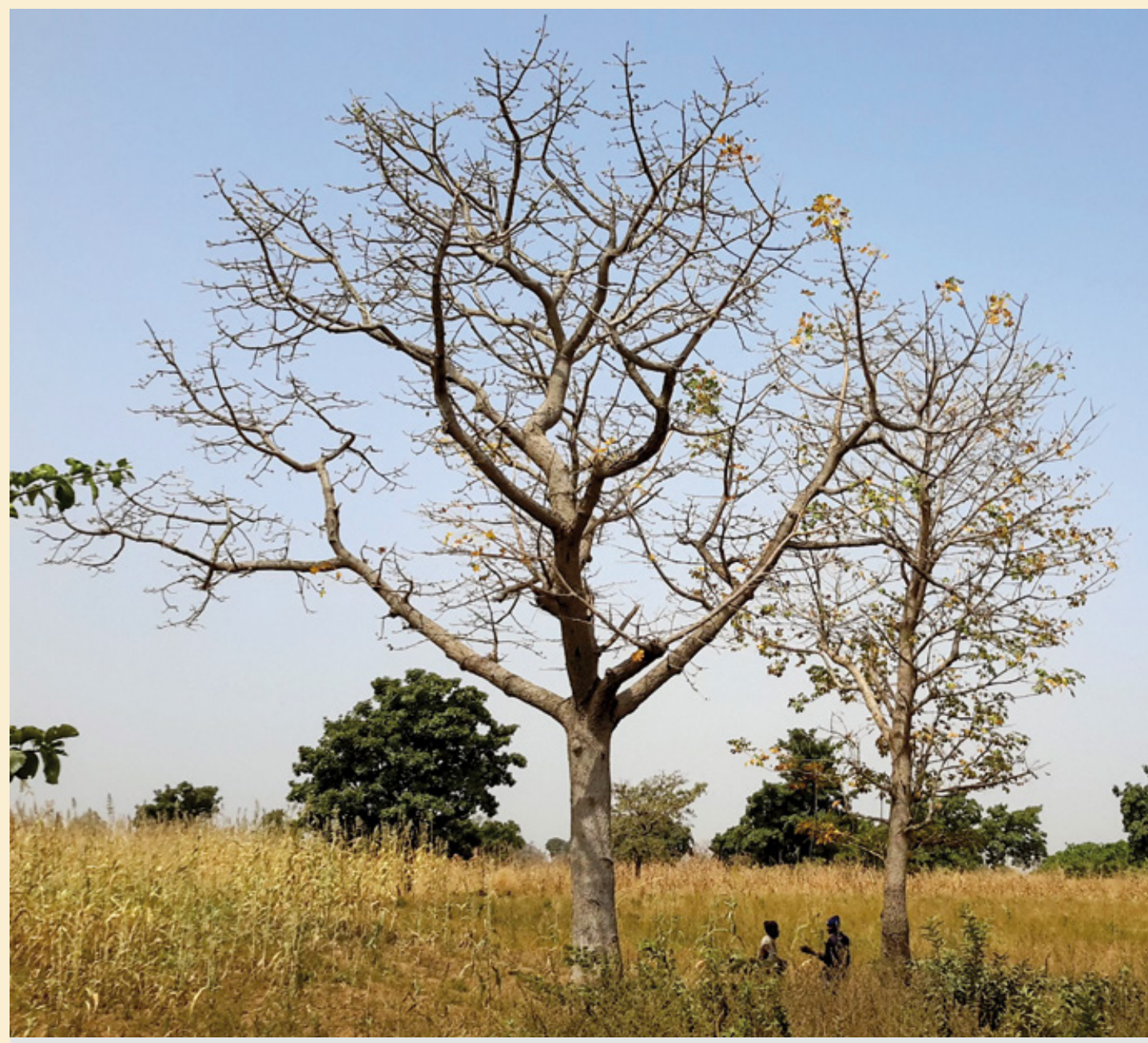

Photo 1.

Bombax costatum en savane dans la zone soudanienne du Bénin.

Photo G. A. Assogba. 


\section{RÉSUMÉ}

\section{USAGES DE BOMBAX COSTATUM (MALVACEAE) DANS LES TERROIRS RIVERAINS DE LA RÉSERVE DE BIOSPHĖRE DE LA PENDJARI, RÉPUBLIQUE DU BÉNIN}

Bombax costatum (faux kapokier) est une espèce des zones soudaniennes peu étudiée et sous-utilisée. Cette étude a évalué les connaissances sur les usages de $B$. costatum en relation avec les facteurs socio-démographiques (âge et sexe) et le groupe socioculturel. À cet effet, 118 entretiens individuels semi-structurés ont été réalisés dans les terroirs riverains de la réserve de biosphère de la Pendjari au Bénin. La fréquence relative de citation, la valeur d'usage rapportée et l'indice d'importance culturelle ont été utilisés pour quantifier les usages et l'importance de l'espèce. Au total, 46 usages ont été recensés et classés en huit catégories d'usages dont les plus citées et ayant les valeurs d'importance culturelle les plus élevées sont les usages alimentaires et médicinaux. Le calice est la partie de plante la plus sollicitée pour les usages alimentaires; il est de surcroît localement commercialisé. Les usages médicinaux impliquent plusieurs organes/parties (calice, feuille, écorce, graine et racine). Les connaissances sur les usages de $B$. costatum variaient significativement entre groupes socioculturels mais pas entre sexe et catégorie d'âges. En effet, les groupes socioculturels Berbas et Gourmantchés, contrairement aux Waamas, Natimbas et Peulhs, possédaient plus de connaissances sur les usages de $B$. costatum et avaient une préférence pour les usages alimentaires. La promotion des usages alimentaires de $B$. costatum peut être envisagée et ainsi servir de point de départ pour sa plus large valorisation. Les études futures devraient toutefois explorer d'autres régions d'occurrence de l'espèce et mettre l'accent sur la valeur nutritionnelle, les revenus générés par la plante, de même que sa propagation en vue de sa valorisation.

Mots-clés : Bombax costatum, connaissance traditionnelle, valeur d'usage ethnobotanique, réserve de biosphère de la Pendjari, Bénin.

\section{ABSTRACT}

\section{USES OF BOMBAX COSTATUM \\ (MALVACEAE) IN LANDS ADJACENT TO THE PENDJARI BIOSPHERE RESERVE IN THE REPUBLIC OF BENIN}

Bombax costatum (the red kapok tree) is native to Africa's Sudanian zone. It has rarely been studied and is most certainly under-used. Our study assessed traditional knowledge on uses of $B$. costatum and its cultural importance in different socio-cultural groups and according to age categories and gender around the Pendjari Biosphere Reserve in the Republic of Benin. We conducted 118 individual semi-structured interviews to make the assessment. Ethnobotanical indices such relative frequency of citation, reported use value and the cultural importance index were applied in order to assess traditional knowledge and the cultural importance of $B$. costatum. A total of 46 uses were identified and grouped into 8 categories. The most frequently cited and most culturally important uses of the species were for food and medicinal purposes. The calyx was the plant part most frequently used, especially for food, while medicinal uses involved several plant parts including the calyx, leaf, bark, seed and root. The calyx is also sold on local markets. Knowledge on the uses of $B$. costatum varied significantly between socio-cultural groups but not according to gender or age categories. Respondents from the Berba and Gourmantché socio-cultural groups were more knowledgeable than the Waama, Natimba and Peulh groups, and also used B. costatum for food most frequently. Promoting the food uses of this species could be considered as a starting point for broadening its use as a resource. However, future studies would need to explore other areas where the species occurs and focus additionally on its nutritional value and potential contribution to household income, as well as on efficient propagation methods to ensure that the species can be used sustainably.

Keywords: Bombax costatum, traditional knowledge, ethnobotanical use value, Pendjari Biosphere Reserve, Republic of Benin.

\section{USOS DE BOMBAX COSTATUM (MALVACEAE) EN LAS TIERRAS COLINDANTES CON LA RESERVA DE LA BIOSFERA DE PENDJARI EN LA REPÚBLICA DE BENÍN}

Bombax costatum es una especie de la zona sudanesa poco estudiada e infrautilizada. Este estudio evaluó los conocimientos sobre los usos de B. costatum y su relación con los factores sociodemográficos (edad y sexo) y el grupo sociocultural. Para ello, se realizaron 118 entrevistas individuales semiestructuradas en las tierras colindantes con la Reserva de la Biosfera de Pendjari en Benín. Se cuantificaron los usos y la importancia de la especie mediante la frecuencia relativa de citación, el valor de uso reportado y el índice de importancia cultural. En total, se identificaron 46 usos que fueron clasificados en 8 categorías. Los usos alimentarios y medicinales fueron los más citados y los que poseían los índices de importancia cultural más altos. El cáliz es la parte de la planta más demandada para los usos alimentarios y, además, se comercializa localmente. Los usos medicinales comprenden varios órganos o partes (cáliz, hoja, corteza, semilla y raíz). El conocimiento de los usos de $B$. costatum variaba significativamente entre grupos socioculturales, pero no entre sexo y categoría de edad. Así pues, los grupos socioculturales Berba y Gourmantché poseían más conocimientos sobre los usos de B. costatum que los Waama, Natimba y Peulh; además, mostraban una preferencia por los usos alimentarios de esta planta. Se podría contemplar un fomento de los usos alimentarios de $B$. costatum como punto de partida para una mayor valorización. No obstante, los futuros estudios deberían explorar otras áreas con presencia de esta especie haciendo hincapié en su valor nutricional, los ingresos generados por la planta y sus modos de propagación con el fin de lograr su valorización.

Palabras clave: Bombax costatum, conocimiento tradicional, valor de uso etnobotánico, Reserva de la Biosfera de Pendjari, Benín. 


\section{Introduction}

Plusieurs populations autochtones à travers le monde dépendent des ressources végétales et emploient leurs connaissances accumulées dans le temps pour en tirer profit et les gérer de façon durable (Sofowora, 2010). Les produits forestiers non ligneux (PFNL) sont des biens d'origine biologique autres que le bois, provenant des forêts, d'autres terrains boisés ou provenant d'arbres hors forêts (FAO, 2001). Ceux-ci sont importants et leur valorisation et leur gestion durable pourraient efficacement contribuer à l'atteinte des Objectifs de Développement Durable (ODD) qui visent, entre autres, à éliminer la pauvreté, la faim et à assurer la sécurité alimentaire dans le monde (Fandohan et al., 2015 ; Lescuyer et al., 2010 ; Ingram et Bongers, 2009). En effet, pour les populations surtout rurales, les PFNL revêtent une importance capitale car ils contribuent aux soins de santé, à l'alimentation, au revenu monétaire et aux autres aspects de leur bien-être (Cavendish, 2000 ; Mahapatra et al., 2005).

Bien que plusieurs espèces pourvoyeuses de ces ressources ont fait et continuent de faire l'objet d'études, plusieurs autres sont insuffisamment connues et documentées (Codjia et al., 2003) dans les pays tropicaux; c'est le cas de Bombax costatum (Belem et al., 2008) (photo 1).

Au Bénin, au cours de cette dernière décennie, de nombreuses études se sont focalisées sur l'importance socioculturelle des plantes endogènes (Dadjo et al., 2012 ; Fandohan et al., 2010 ; Ekué et al., 2010 ; Koura et al., 2011 ; Gouwakinnou et al., 2011). Toutefois, aucune d'entre elles n'a décrit les usages de $B$. costatum.

De la famille des malvacées, $B$. costatum est une espèce commune qui est exploitée par les populations rurales mais est localement peu abondante dans les zones guinéo-soudanienne et soudanienne du Bénin (Arbonnier, 2002 ; Oyen, 2011).

L'intérêt qu'accordent la communauté scientifique et les décideurs politiques à cette espèce est encore assez faible (Belem et al., 2008 ; Ouédraogo et al., 2014). Dans une étude récente, Belem et al. (2008) ont montré qu'au Burkina Faso l'espèce est essentiellement utilisée dans l'alimentation humaine, dans les soins de santé, dans l'artisanat, dans les rites culturels et religieux. L'évaluation de l'importance socioculturelle et économique de telles ressources à travers leurs aires d'occurrence constitue un des prérequis à l'élaboration de stratégies globales d'utilisation durable. Elle aiderait entre autres à : savoir comment B. costatum contribue au bien-être des communautés rurales; estimer sa valeur écologique et son importance dans le maintien de l'intégrité des écosystèmes ; savoir comment l'espèce est intégrée dans les cultures et rituels de chaque peuple ; enfin, à envisager une valorisation économique.

Ainsi, la présente étude vise à décrire les usages de B. costatum et évaluer son importance culturelle pour les différents groupes socioculturels observés dans une de ses zones majeures d'occurrence au Bénin, la réserve de biosphère de la Pendjari. En admettant que les usages et le degré de connaissance des plantes dépendent de la culture, des us et coutumes des peuples, de l'âge et du genre (Camou-Guerrero et al., 2008 ; Gouwakinnou et al., 2011), cette étude a comparé les connaissances sur $B$. costatum entre groupes socioculturels, entre catégories d'âges et entre sexes.

\section{Matériel et méthodes}

\section{Milieu d'étude et choix des localités d'enquête}

L'étude a été réalisée dans 12 villages des terroirs riverains de la réserve de biosphère de la Pendjari (RBP), notamment les communes de Tanguiéta et de Matéri (figure 1) où l'espèce est naturellement rencontrée. Cette partie de la RBP dénommée zone d'occupation contrôlée (ZOC) se situe géographiquement entre les latitudes $10^{\circ} 30^{\prime}-11^{\circ} 30^{\prime}$ Nord et les longitudes $0^{\circ} 50^{\prime}-2^{\circ} 00^{\prime}$ Est (figure 1). Cette zone est caractérisée par la présence de la chaîne montagneuse de l'Atacora. Elle jouit d'un climat de type soudanien avec un régime saisonnier unimodal. La pluviométrie annuelle oscille entre $900 \mathrm{~mm}$ et $1000 \mathrm{~mm}$ (Adomou, 2005). La température moyenne mensuelle varie de 30 à $34^{\circ} \mathrm{C}$. La saison sèche s'étend sur une période d'environ sept mois. La végétation est dominée par des savanes boisées et des forêts claires par endroits. La ZOC subit une forte pression anthropique

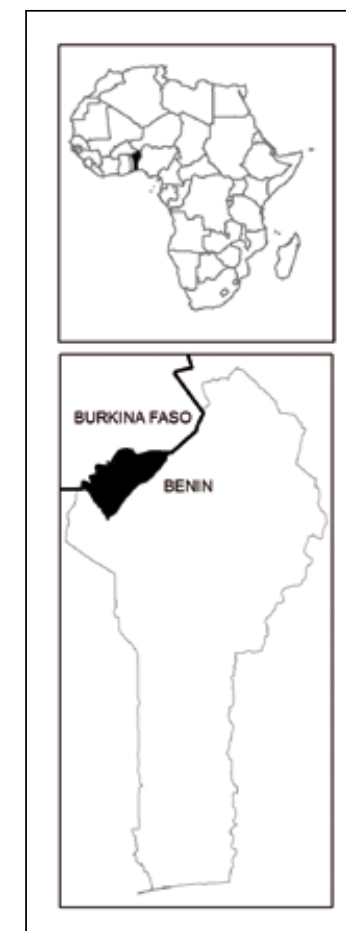

Réalisation : Assogba, 2016.

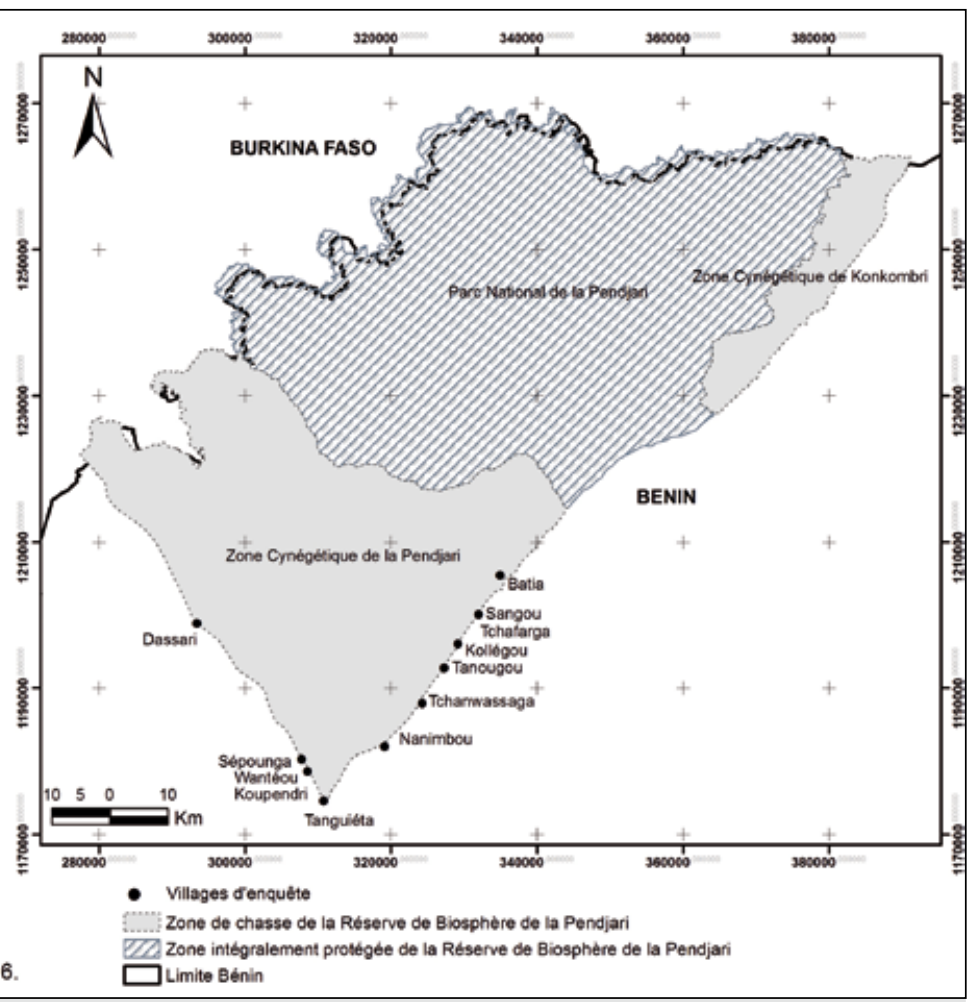

Figure 1.

Localisation de la réserve de biosphère de la Pendjari et des localités d'étude au Bénin. 
due à l'accroissement de la population humaine dans les communes de Matéri (111 003 habitants dont 51,48 \% de femmes) et de Tanguiéta (73 731 habitants dont 51,04\% de femmes) (RGPH4, 2015). Les principaux groupes socioculturels qui vivent dans cette zone sont les Berbas, les Gourmantchés, les Waamas, les Peulhs et les Natimbas. Ces groupes font partie des populations les plus pauvres du Bénin, vivant en dessous de 1 USD par jour (CENAGREF, 2009). Leurs principales activités sont l'agriculture, la chasse, l'élevage (volaille, petits ruminants et bovins) et la pêche (CENAGREF, 2009). Ces populations sont fortement dépendantes des ressources biologiques de la RBP et plus particulièrement des produits forestiers non ligneux dans l'alimentation, la santé, les infrastructures et les soins de santé (Vodouhê et al., 2009).

\section{Échantillonnage}

La sélection des enquêtés s'est faite de façon aléatoire et simple. À cet effet, un sondage exploratoire sur un échantillon de 50 personnes prises au hasard dans chacune des deux communes a permis de déterminer la proportion ( $p$ ) de ceux qui connaissent au moins un usage de B. costatum. La détermination du nombre d'enquêtés a alors été faite en utilisant la formule de Dagnelie (1998) :

$$
\mathrm{n}=\frac{\mathrm{U}_{1-\alpha / 2}^{2} \times \mathrm{p}(1-\mathrm{p})}{\mathrm{d}^{2}}
$$

où $n$ représente la taille de l'échantillon global ; $U_{1-\alpha / 2}$ est la valeur de la variable normale aléatoire pour une valeur de probabilité de $\alpha ; U_{1-\alpha / 2}=1,96$ si $\alpha=0,05$; p est la proportion d'individus de $B$. costatum; d est l'erreur marginale.

L'enquête exploratoire a révélé qu'environ $42 \%$ et $58 \%$ des personnes interrogées connaissent et utilisent l'espèce dans les communes de Matéri et de Tanguiéta, respectivement. Ainsi, pour une marge d'erreur de $8 \%$, la taille de l'échantillon correspondait à 50 et 68 personnes respectivement dans les communes de Matéri et de Tanguiéta ; 50 et 68 personnes ont été sélectionnées et interviewées dans ces deux communes en fonction de leur consentement libre à participer à l'enquête. Ces personnes ont été choisies au hasard sur des listes des personnes (établies avec les chefs de village et/ou avec des informateurs clés identifiés par les chefs de village) vivant dans les localités d’étude.

\section{Enquêtes ethnobotaniques}

Les données ont été collectées à travers des entretiens individuels semi-structurés et des observations directes. Le questionnaire de base était structuré en deux parties. La première partie vise à renseigner l'âge, le sexe, le groupe socioculturel de l'enquêté ainsi que sa profession ou sa principale activité. La seconde partie concerne, d'une part, les connaissances de l'enquêté sur les usages faits de l'espèce, ses parties ou organes et, d'autre part, l'importance culturelle de la plante et de ses parties ou organes. Les catégories d'usages utilisées à cet effet sont adaptées de Fandohan et al. (2010) et de Tardio et Pardo-De-Santayana (2008) : alimentaire, artisanat, bois de service, cultuel, écologique, matériel, médecine et socioculturel.

L'évaluation de l'importance culturelle de la plante et de ses parties/organes a été menée en utilisant une grille de score d'importance à quatre niveaux (Belem et al., 2008). En effet, dans un premier temps, il a été demandé à chaque enquêté d'attribuer un score d'importance à chaque catégorie d'usages pour la plante entière. Dans un second temps, le même exercice a été fait pour chaque catégorie d'usages, mais cette fois par partie/organe de plante. Les scores variaient de 0 à 1,5 . Le score 0 correspondait à une catégorie d'usages non importante ; 0,5 était attribué à une catégorie d'usages moyennement importante ; 1 était affecté à une catégorie d'usages importante ; 1,5 était le score maximal et correspondait à une catégorie d'usages très importante.

\section{Traitement et analyses statistiques des données}

Les 118 personnes enquêtées appartenaient à cinq groupes socioculturels (Gourmantchés, Berbas, Waamas, Natimbas et Peulhs). Elles ont été réparties suivant le sexe et la classe d'âge. La répartition suivant la classe d'âge a suivi celle de Assogbadjo et al. (2008) comme suit : les jeunes (âge $\leq 30$ ans) ; les adultes (30< âge <60); les vieux (âge $\geq 60$ ans). Du fait de la faible représentativité des groupes socioculturels Waama, Natimba et Peulh dans l'échantillon, pouvant biaiser les estimations, ces trois groupes socioculturels ont été fusionnés en « autres groupes socioculturels » (tableau I).

Quatre indices ethnobotaniques, la fréquence relative de citation, la valeur d'usage totale rapportée, la valeur d'usage rapportée par organe et l'indice d'importance

Tableau I.

Effectif des personnes enquêtées selon le groupe socioculturel, la classe d'âge et le sexe.

\begin{tabular}{|c|c|c|c|c|c|c|c|}
\hline Catégories & Gou & chés & & & Autres & oculturels* & Total \\
\hline socioculturelles & H & $\mathbf{F}$ & $\mathbf{H}$ & $\mathbf{F}$ & H & $\mathbf{F}$ & \\
\hline Jeunes (âge < 30 ans) & 5 & 6 & 4 & 6 & 4 & 4 & 29 \\
\hline Adultes (30< âge <60) & 7 & 9 & 9 & 15 & 8 & 8 & 56 \\
\hline Vieux (âge $\geq 60$ ans) & 4 & 4 & 7 & 6 & 6 & 6 & 33 \\
\hline Total & 16 & 19 & 20 & 27 & 18 & 18 & 118 \\
\hline
\end{tabular}

* Ensemble formé par les Waamas, Natimbas et Peulhs; $\mathrm{H}=$ homme ; $\mathrm{F}=$ femme. 
culturelle, ont été calculés pour évaluer respectivement les connaissances et le consensus des enquêtés sur ces connaissances, les usages et l'importance culturelle de B. costatum.

La fréquence relative de citation (Relative frequency of citation, FRC) se réfère au nombre de fois que les enquêtés d'un groupe social ont cité un usage spécifique donné, rapporté au nombre total d'enquêtés de ce groupe (Tardio et Pardo-De-Santayana, 2008 ; Honfo et al., 2015) :

$F R C=\frac{n}{N} \times 100$

où $\mathrm{FRC}$ est la fréquence relative de citation exprimée en pourcentage ; n le nombre d'enquêtés ayant cité un usage donné de $B$. costatum ; N le nombre total d'enquêtés.

La FRC a permis d'ordonner les usages spécifiques des parties/organes de $B$. costatum. Des valeurs élevées de la FRC pour un usage spécifique traduisent généralement un consensus pour cet usage de la partie/organe au sein de la communauté.

La valeur d'usage totale rapportée (VUR) (Gomez-Beloz, 2002) pour un groupe est le nombre total moyen d'usages spécifiques rapportés pour $B$. costatum dans ce groupe, exprimé en usages spécifiques par enquêté :

$\operatorname{VUR}=\frac{\sum_{\mathrm{i}=1}^{\mathrm{N}} \mathrm{VUR}_{\mathrm{i}}}{\mathrm{N}}$

où VURi est le nombre total d'usages spécifiques rapportés par l'enquêté i du groupe ; N est le nombre total d'enquêtés pour le groupe social considéré.

Cet indice a permis de mesurer et de comparer les connaissances des enquêtés entre les différents groupes socioculturels, catégories d'âges et sexes. Les groupes présentant des VUR élevées sont ceux possédant globalement plus de connaissances sur les usages de la plante. Les valeurs de la VUR étant des données de comptage ; elles ont été soumises à un modèle linéaire généralisé de la famille de Poisson pour tester l'effet du groupe socioculturel, de l'âge et du sexe sur sa variation. Le modèle saturé (tous les effets principaux et interactions possibles) a été d'abord spécifié. Ensuite, une simplification du modèle a été opérée jusqu'à la sélection du modèle parcimonieux (modèle contenant le moins possible de facteurs) Johnson et Omland, 2004). Cette simplification a été basée sur la valeur du critère d'information d'Akaike corrigé pour les petites tailles d'échantillon (Akaike, 1973 ; Johnson et Omland, 2004). Les analyses ont été réalisées dans le logiciel $R$ version 3.2 ( $R$ Core Team, 2016).

La valeur d'usage rapportée par organe $\left(V U R_{\text {org }}\right)$ (Gomez-Beloz, 2002) est le nombre moyen d'usages rapportés pour chaque organe de $B$. costatum, exprimé en usages spécifiques par enquêté :

$\operatorname{VURorg}_{x}=\frac{\sum_{i=1}^{N} \operatorname{VUR}_{x i}}{N}$ où VURorg ${ }_{x}$ est la valeur d'usage rapportée pour la partie/ organe $x ; V R_{x i}$ est le nombre total d'usages spécifiques de la partie/organe $x$ mentionné par l'enquêté i du groupe considéré ; $\mathrm{N}$ est le nombre total d'enquêtés du groupe.

Cet indice a permis d'évaluer le nombre d'usages connus par partie/organe de la plante. Les parties/organes présentant des VUR org élevées sont ceux ayant le plus grand nombre d'usages, et donc probablement les plus souvent sollicités par les populations. Un modèle linéaire généralisé de la famille de Poisson a été utilisé pour tester la significativité des différences des valeurs de VUR org entre parties/organes de plante.

L'indice d'importance culturelle (IC) (Houehanou et al., 2011) désigne, d'une part, la valeur culturelle allouée à la plante et, d'autre part, celle rattachée aux parties/organes de la plante par les populations locales. La valeur de l'IC a été calculée par catégorie d'usages $\left(\mathrm{IC}_{\mathrm{CU}}\right)$ pour chaque groupe socioculturel d'une part et pour chaque partie/organe de la plante d'autre part. Dans le premier cas, l'IC permet de mettre en exergue la catégorie d'usages la plus importante selon les groupes socioculturels et, dans le second cas, la catégorie d'usages la plus importante selon la partie/organe de la plante. La valeur de IC a été par ailleurs calculée par partie/organe $\left(\mathrm{IC}_{\text {Org }}\right)$ et pour chaque groupe socioculturel afin de mettre en exergue les parties/organes de plantes les plus importants pour chaque groupe socioculturel.

Pour chaque groupe socioculturel (respectivement chaque partie/organe), l'importance culturelle d'une catégorie d'usages quelconque $\mathrm{k}$ a été calculée en utilisant la formule ci-dessous :

$I C_{C_{k}}=\frac{\sum_{i=1}^{N} S_{C U_{k i}}}{N}$

où $S_{\mathrm{CU}_{\mathrm{ki}}}$ est le score attribué par l'enquêté i du groupe socioculturel considéré à la catégorie d'usages $\mathrm{k}\left(\mathrm{CU}_{\mathrm{k}}\right)$ de la plante ; N désigne le nombre total d'enquêtés.

L'indice d'importance culturelle de chaque partie/ organe de plante pour un groupe socioculturel donné a été calculé en utilisant la formule ci-dessous :

$I C_{\mathrm{PP} / \mathrm{Org}_{\mathrm{m}}}=\frac{\sum_{\mathrm{i}}^{\mathrm{N}} \sum_{\mathrm{k}=1}^{\mathrm{K}} \mathrm{S}_{\mathrm{PP} / \mathrm{Org}_{\mathrm{m}}-\mathrm{CU}_{\mathrm{ki}}}}{\mathrm{N}}$

où $\mathrm{S}_{\mathrm{Pp} / \mathrm{Org}_{\mathrm{m}}-\mathrm{Cu}_{\mathrm{ki}}}$ est le score attribué par l'enquêté i du groupe socioculturel considéré à la partie de plante/organe $\mathrm{m}$ dans la catégorie d'usages $\mathrm{k}\left(\mathrm{CU}_{\mathrm{k}}\right)$ de la plante ; $\mathrm{K}$ désigne le nombre total de catégories d'usages ( $K=8$ dans la présente étude) ; $\mathrm{N}$ désigne le nombre total d'enquêtés du groupe socioculturel considéré.

Les valeurs de IC étant des scores moyens, elles ont été soumises à un test de Kruskal-Wallis (Dytham, 2011) pour comparer l'importance culturelle de chaque catégorie d'usages de $B$. costatum entre groupes socioculturels et entre parties de plante ou organes, d'une part, et l'importance culturelle de chaque partie de plante ou organe entre groupes socioculturels, d'autre part. Des diagrammes en radar ont été utilisés pour illustrer les résultats. 


\section{Résultats}

\section{Appellations locales de $B$. costatum}

Le tableau II résume les appellations de $B$. costatum et leur signification selon chaque groupe socioculturel. Les appellations locales de $B$. costatum diffèrent d'un groupe socioculturel à un autre. Toutefois, les significations restent semblables. B. costatum est en effet soit appelé plante à fleurs rouges (photo 2), soit arbre léger.

\section{Diversité des connaissances sur les usages de $B$. costatum}

Le tableau III présente la diversité des usages de $B$. costatum. Il en ressort que les populations des terroirs riverains de la réserve de biosphère de la Pendjari connaissent en général 46 usages de l'espèce. Les enquêtés qui ont affirmé que l'espèce est principalement utilisée pour l'alimentation humaine (sauce légume, sauce gluante, huile comestible et

\begin{tabular}{|c|c|c|}
\hline \multicolumn{3}{|c|}{$\begin{array}{l}\text { Tableau II. } \\
\text { Appellation locale et signification de Bombax costatum } \\
\text { selon les groupes socioculturels. }\end{array}$} \\
\hline Groupe socioculturel & Appellation & Signification \\
\hline Gourmantchés & Boufouébou & Arbre léger \\
\hline Waamas & Fokobou/Fôguéré & Arbre à fleurs rouges \\
\hline Berbas & Fangque & Arbre à fleurs rouges \\
\hline Peulhs & Koukoii & Arbre à fleurs rouges \\
\hline Natimbas & Tchéhobou & Arbre léger \\
\hline
\end{tabular}

boisson) représentent $90 \%$ de l'échantillon d'étude alors que seulement $6 \%$ ont rapporté l'usage de la plante dans l'alimentation animale comme fourrage. De plus, les usages médicinaux de $B$. costatum dans le traitement des maladies non infectieuses ont été mentionnés par $87 \%$ des enquêtés. Les usages cultuels et écologiques de $B$. costatum sont également non négligeables (respectivement $24 \%$ et $28 \%$ des enquêtés). Entre autres, $B$. costatum est utilisé dans l'artisanat, comme bois de service, et aussi comme matériel de fabrication de divers objets utiles.

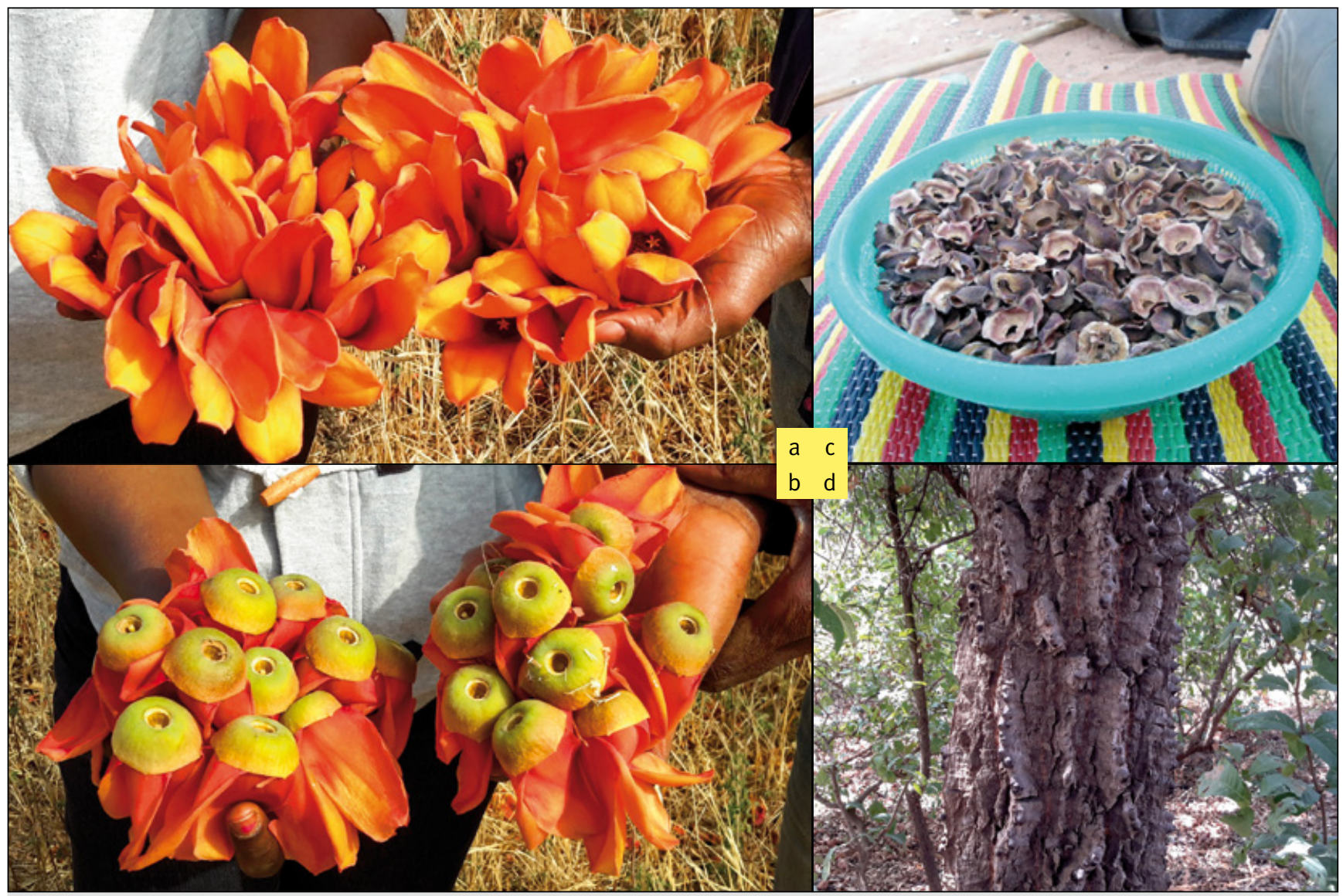

Photo 2.

Quelques organes/parties de Bombax costatum utilisés par les populations locales des terroirs riverains de la réserve de biosphère de la Pendjari : a) fleurs, b) calices frais, c) calices secs, d) écorces. Photo G. A. Assogba. 
Tableau III.

Usages spécifiques de Bombax costatum et leur importance (+++ : très important ; ++ : moyennement important ; + : peu important) et fréquences relatives de citation.

\begin{tabular}{|c|c|c|c|c|}
\hline $\begin{array}{l}\text { Catégorie } \\
\text { d'usages }\end{array}$ & $\begin{array}{l}\text { Organe ou } \\
\text { partie de plante }\end{array}$ & Usages spécifiques & Importance & FRC \\
\hline \multirow[t]{8}{*}{ Alimentaire } & \multirow[t]{4}{*}{ Feuille } & Sauce légume & +++ & \multirow[t]{8}{*}{$90 \%$} \\
\hline & & Fourrage pour bétail & +++ & \\
\hline & & Boisson & ++ & \\
\hline & & Stérilisation de l'eau & ++ & \\
\hline & Graine & Huile comestible & ++ & \\
\hline & Fleur & Sauce gluante & ++ & \\
\hline & \multirow[t]{2}{*}{ Calice } & Sauce gluante & +++ & \\
\hline & & Sauce légume & +++ & \\
\hline \multirow[t]{3}{*}{ Artisanat } & Fleur & Teinture & + & \multirow[t]{3}{*}{$11 \%$} \\
\hline & \multirow[t]{2}{*}{ Tige } & Sculpture & ++ & \\
\hline & & Fabrication d'objets d'art & ++ & \\
\hline \multirow[t]{3}{*}{ Bois de service } & \multirow[t]{3}{*}{ Tige } & Construction de bâtiments & ++ & \multirow[t]{3}{*}{$9 \%$} \\
\hline & & Fabrication de meubles & ++ & \\
\hline & & Coffrage & +++ & \\
\hline \multirow[t]{10}{*}{ Cultuel } & \multirow[t]{2}{*}{ Feuille } & Composition des recettes de foudre & ++ & \multirow[t]{10}{*}{$24 \%$} \\
\hline & & Convulsion & ++ & \\
\hline & \multirow[t]{3}{*}{ Écorce } & Convulsion & ++ & \\
\hline & & Composition des poudres chimiques & ++ & \\
\hline & & Composition des recettes de guérison de la folie & ++ & \\
\hline & \multirow[t]{5}{*}{ Racine } & Composition des recettes de foudre & ++ & \\
\hline & & Composition des recettes d'attraction de chance & ++ & \\
\hline & & Convulsion & ++ & \\
\hline & & Composition des poudres chimiques & ++ & \\
\hline & & Composition des recettes de guérison de la folie & ++ & \\
\hline \multirow[t]{2}{*}{ Écologique } & Fleur & Nid d’essaim d’abeilles (plantes mellifères) & ++ & \multirow[t]{2}{*}{$28 \%$} \\
\hline & Feuille & Nid d’essaim d’abeilles (plantes mellifères) & ++ & \\
\hline \multirow[t]{8}{*}{ Matériel } & Graine & Fabrication de matelas & ++ & \multirow[t]{8}{*}{$44 \%$} \\
\hline & \multirow[t]{7}{*}{ Tige } & Fabrication de fournitures bureautiques & + & \\
\hline & & Fabrication de pirogues & ++ & \\
\hline & & Fabrication de bâtons de berger & +++ & \\
\hline & & Fabrication d'ustensiles de cuisine & ++ & \\
\hline & & Fabrication d'abreuvoirs et de mangeoires pour les animaux & ++ & \\
\hline & & Fabrication de tambours & +++ & \\
\hline & & Flotteur d'hameçon & +++ & \\
\hline \multirow[t]{15}{*}{ Médecine } & Feuille & Paludisme & +++ & $87 \%$ \\
\hline & Écorce & Fièvre & +++ & \\
\hline & & Dermatoses & +++ & \\
\hline & & Courbature & +++ & \\
\hline & & Abcès & ++ & \\
\hline & & Mitose & ++ & \\
\hline & & Fldèmes & +++ & \\
\hline & & Hernie & + & \\
\hline & & Épilepsie & +++ & \\
\hline & Graine & Paludisme & +++ & \\
\hline & & Fabrication d'huile de massage & ++ & \\
\hline & & Vermifuge pour bétail & +++ & \\
\hline & Racine & Accouchement difficile & ++ & \\
\hline & & Hémorroïdes & ++ & \\
\hline & & Inflammation des pieds & ++ & \\
\hline Socioculturel & Écorce & Rituels (cicatrisation des balafres) & ++ & $14 \%$ \\
\hline & Tige & Instruments de musique traditionnelle & +++ & \\
\hline
\end{tabular}




\section{Facteurs socio-démographiques influençant le niveau de connaissance des usages de $B$. costatum}

Le tableau IV résume les différents modèles testés. Le modèle incluant seulement le groupe socioculturel est celui ayant la plus faible valeur de l'AICc et classé modèle le plus parcimonieux (tableau IV). En d'autres termes, parmi les facteurs testés, seul le groupe socioculturel déterminait les variations du niveau des connaissances des usages de $B$. costatum. En effet, la valeur d'usage totale de B. costatum pour les Gourmantchés était significativement plus élevée que celle des " autres groupes socioculturels » $(P=$ 0,049 ; tableau V). Par contre, bien que la valeur d'usage totale de $B$. costatum pour les Berbas soit plus élevée que celle des " autres groupes socioculturels ", la différence n'était pas significative $(P=0,094$; tableau $V)$.
La figure 2 illustre bien que la valeur d'usage totale (moyenne \pm erreur type) de $B$. costatum était similaire entre les Gourmantchés $(3,71 \pm 0,26)$ et les Berbas $(3,51 \pm 0,24)$ et plus faible pour les " autres groupes socioculturels " $(2,67 \pm 0,34)$.

\section{Valeur d'usage des parties/organes de B. costatum}

La valeur d'usage de $B$. costatum variait significativement entre parties/organes de la plante (tableau VI). Le calice $(0,79 \pm 0,04)$ est la partie/organe de $B$. costatum la plus utilisée, suivie de la jeune tige $(0,73 \pm 0,04)$ et de l'écorce $(0,66 \pm 0,06)$ (tableau $\mathrm{VI}$ et figure 3$)$. La partie/ organe la moins utilisée est la graine $(0,17 \pm 0,04)$ (tableau VI et figure 3).

\begin{abstract}
Tableau IV.
Variation de la valeur d'usage de Bombax costatum suivant les caractéristiques des enquêtés : résultats de la sélection des modèles relatifs à l'effet des facteurs socio-démographiques (âge, sexe, groupe socioculturel) sur le niveau de connaissance des usages de Bombax costatum.
\end{abstract}

\section{Les modèles candidats}

GSC

Sexe + GSC

Sexe + GSC + Sexe $\times$ GSC

$\hat{A} g e+$ Sexe + GSC + Sexe $\times$ GSC

Âge + Sexe + GSC + Sexe $\times$ Âge + GSC $\times$ Sexe

$\hat{A} g e+$ Sexe + GSC + Sexe $\times \hat{A} g e+G S C \times \hat{A} g e+G S C \times$ Sexe

GSC : groupe socioculturel ; Âge : catégorie d'âge.

Les modèles ont été classés suivant les valeurs du critère d'information d'Akaike corrigé pour les petites tailles d'échantillons (AICC). Le $\triangle \mathrm{AICC}$ d'un modèle est la différence entre l'AICc de ce modèle et celui du modèle parcimonieux, le premier modèle étant le modèle le plus parcimonieux.

\section{Tableau V.}

Estimations pour le modèle parcimonieux relatif à l'effet des facteurs sociodémographiques (âge, sexe, groupe socioculturel) sur le niveau de connaissance des usages de Bombax costatum.

\begin{tabular}{|c|c|c|c|c|}
\hline & Estimate & Erreur type & Valeur de Z & Prob. (>|z|) \\
\hline Intercept & 0,981 & 0,144 & 6,796 & $<0,001^{\star \star \star}$ \\
\hline Berbas & 0,275 & 0,164 & 1,677 & $0,094^{\text {ns }}$ \\
\hline Gourmantchés & 0,331 & 0,169 & 1,962 & $0,049^{\star}$ \\
\hline
\end{tabular}

\section{Importance culturelle de B. costatum}

L'importance culturelle de $B$. costatum variait entre les groupes socioculturels seulement pour les catégories d'usages alimentaires et les usages comme matériel ( $P<0,05$; tableau VII). L'importance des autres catégories d'usages était statistiquement similaire entre les groupes socioculturels. La figure 4 révèle que les Berbas accordaient une plus grande importance aux usages alimentaires, médicinaux et matériels de $B$. costatum, comparés aux autres groupes socioculturels. Bien que la différence soit non significative, l'importance des avantages écologiques de $B$. costatum (notamment le fait qu'elle soit une plante mellifère) était relativement plus marquée chez les Gourmantchés (figure 4). Les usages de $B$. costatum comme bois de service sont en général peu importants pour les enquêtés (figure 4).

\section{Importance culturelle des} parties/organes de $B$. costatum

L'importance culturelle de chaque partie/organe de plante de $B$. costatum diffère significativement entre catégories d'usages (tableau VIII). Toutefois, l'importance culturelle du calice, de la racine et de la fleur ne diffère pas entre groupes socioculturels (tableau IX). 
Tableau VI.

Variation de la valeur d'usage de Bombax costatum en fonction de ses parties de plante/organes : résultats des estimations du modèle de Poisson.

\begin{tabular}{|c|c|c|c|c|}
\hline & Estimate & Erreur type & Valeur de Z & Prob. (>|z|) \\
\hline Intercept & $-0,236$ & 0,113 & $-2,095$ & $0,036^{\star}$ \\
\hline Écorce & $-0,180$ & 0,167 & $-1,078$ & $0,281^{\mathrm{ns}}$ \\
\hline Feuille & $-0,935$ & 0,212 & $-4,414$ & $\left\langle 0,001^{\star \star \star}\right.$ \\
\hline Fleur & $-0,843$ & 0,205 & $-4,110$ & $\left\langle 0,001^{\star \star \star}\right.$ \\
\hline Graine & $-1,536$ & 0,267 & $-5,747$ & $\left\langle 0,001^{\star \star \star}\right.$ \\
\hline Jeune tige & $-0,079$ & 0,162 & $-0,487$ & $0,627^{\text {ns }}$ \\
\hline Racine & $-0,632$ & 0,191 & $-3,308$ & $0,001^{* * *}$ \\
\hline
\end{tabular}

Codes du niveau de significativité : $0={ }^{* \star \star} ; 0,001={ }^{* \star} ; 0,01={ }^{*} ;>0,05=\mathrm{ns}$.

Niveaux du facteur partie/organe de plante : calice, écorce, feuille, fleur, graine, jeune tige, racine.

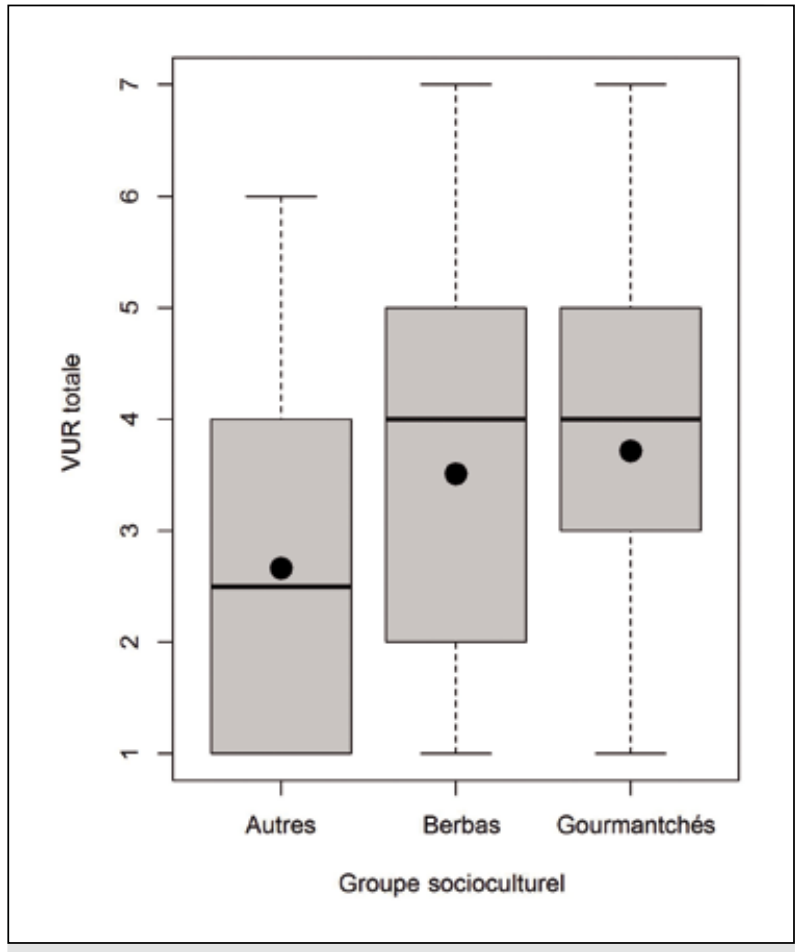

Figure 2.

Boîtes à moustache de la valeur d'usage totale de Bombax costatum selon les groupes socioculturels. Les points noirs indiquent les valeurs moyennes.

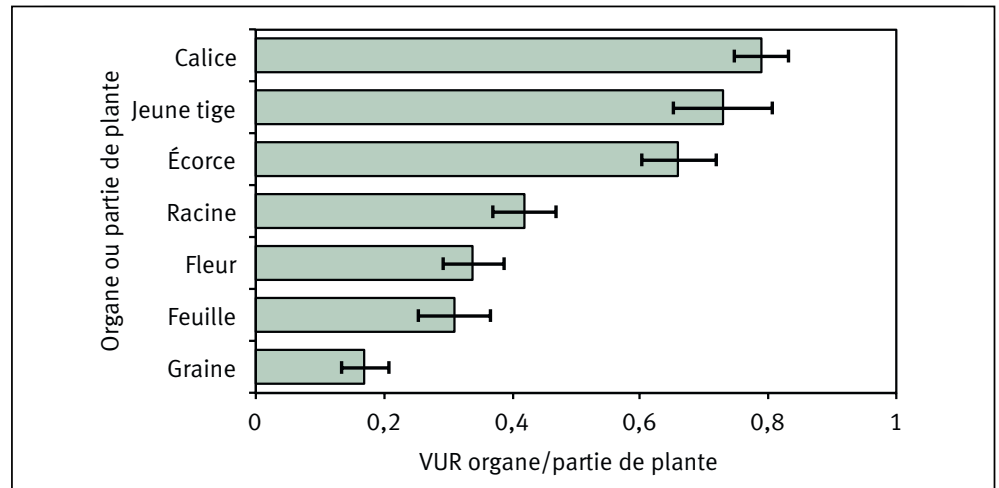

Figure 3.

Diagramme en barre de la valeur d'usage des organes de Bombax costatum.

Tableau VII.

Variation de l'importance culturelle des catégories d'usages de Bombax costatum entre groupes socioculturels : résultats du test de Kruskal-Wallis.

\begin{tabular}{|c|c|c|c|}
\hline Catégories d'usages & ddl & Statistique H & Prob. \\
\hline Alimentation & 2 & 6,46 & $0,039^{*}$ \\
\hline Cultuel & 2 & 1,30 & $0,519^{\text {ns }}$ \\
\hline Médecine & 2 & 4,45 & $0,108^{\text {ns }}$ \\
\hline Socioculturel & 2 & 0,94 & $0,566^{\text {ns }}$ \\
\hline Écologie & 2 & 0,40 & $0,804^{\mathrm{ns}}$ \\
\hline Matériel & 2 & 7,16 & $0,008^{\star *}$ \\
\hline Artisanat & 2 & 1,37 & $0,471^{\text {ns }}$ \\
\hline Bois de service & 2 & 3,35 & $0,111^{\text {ns }}$ \\
\hline
\end{tabular}


Tableau VIII.

Variation de l'importance culturelle de Bombax costatum en fonction de ses organes/parties : moyenne et erreur type de l'indice d'importance culturelle et résultats du test de Kruskal-Wallis.

\begin{tabular}{|c|c|c|c|c|c|c|c|c|}
\hline & Alimentation & Cultuel & Médicinal & Écologie & Socioculturel & Matériel & Artisanat & Bois de service \\
\hline $\begin{array}{l}\text { Organes ou } \\
\text { parties de plante } \\
\text { Calice }\end{array}$ & $1,2 \pm 0,04$ & $0,0 \pm 0,00$ & $0,0 \pm 0,00$ & $0,0 \pm 0,00$ & $0,0 \pm 0,00$ & $0,0 \pm 0,00$ & $0,0 \pm 0,00$ & $0,0 \pm 0,00$ \\
\hline Écorce & $0,0 \pm 0,00$ & $0,6 \pm 0,02$ & $0,9 \pm 0,06$ & $0,0 \pm 0,00$ & $0,0 \pm 0,02$ & $0,0 \pm 0,00$ & $0,0 \pm 0,00$ & $0,0 \pm 0,00$ \\
\hline Feuille & $0,4 \pm 0,03$ & $0,3 \pm 0,02$ & $0,5 \pm 0,05$ & $0,0 \pm 0,01$ & $0,0 \pm 0,00$ & $0,0 \pm 0,00$ & $0,0 \pm 0,00$ & $0,0 \pm 0,00$ \\
\hline Fleur & $0,5 \pm 0,03$ & $0,0 \pm 0,00$ & $0,0 \pm 0,00$ & $0,3 \pm 0,04$ & $0,0 \pm 0,00$ & $0,0 \pm 0,00$ & $0,1 \pm 0,03$ & $0,0 \pm 0,00$ \\
\hline Graine & $0,0 \pm 0,02$ & $0,0 \pm 0,00$ & $0,4 \pm 0,03$ & $0,0 \pm 0,00$ & $0,0 \pm 0,00$ & $0,0 \pm 0,01$ & $0,0 \pm 0,00$ & $0,0 \pm 0,00$ \\
\hline Jeune tige & $0,0 \pm 0,00$ & $0,0 \pm 0,00$ & $0,0 \pm 0,00$ & $0,0 \pm 0,00$ & $0,5 \pm 0,04$ & $0,8 \pm 0,04$ & $0,0 \pm 0,02$ & $0,6 \pm 0,03$ \\
\hline Racine & $0,0 \pm 0,00$ & $0,4 \pm 0,03$ & $0,6 \pm 0,05$ & $0,0 \pm 0,00$ & $0,0 \pm 0,00$ & $0,0 \pm 0,00$ & $0,0 \pm 0,00$ & $0,0 \pm 0,00$ \\
\hline $\begin{array}{l}\text { Résultats du test } \\
\text { de Kruskal-Wallis } \\
\text { ddl }\end{array}$ & 6 & 6 & 6 & 6 & 6 & 6 & 6 & 6 \\
\hline Statistique $\mathrm{H}$ & 239,59 & 43,49 & 167,10 & 69,89 & 57,82 & 94,56 & 28,38 & 31,13 \\
\hline Prob. & $<0,001$ & $<0,001$ & $<0,001$ & $<0,001$ & $<0,001$ & $<0,001$ & $<0,001$ & $<0,001$ \\
\hline
\end{tabular}

H : statistique de Kruskal-Wallis ; ddl : degré de liberté ; Prob. : probabilité liée au test statistique.

En effet, le calice était culturellement plus important pour les usages alimentaires, l'écorce et la racine pour les usages culturels, l'écorce et la feuille pour les usages médicinaux, la fleur pour ses avantages écologiques et la jeune tige pour les usages socioculturels, comme matériel et bois de service (tableau VIII).

La figure 5 présente l'importance culturelle des parties/organes de $B$. costatum en fonction des groupes socioculturels. L'écorce, la feuille et la jeune tige ont une importance culturelle plus grande pour les Gourmantchés et les « autres groupes

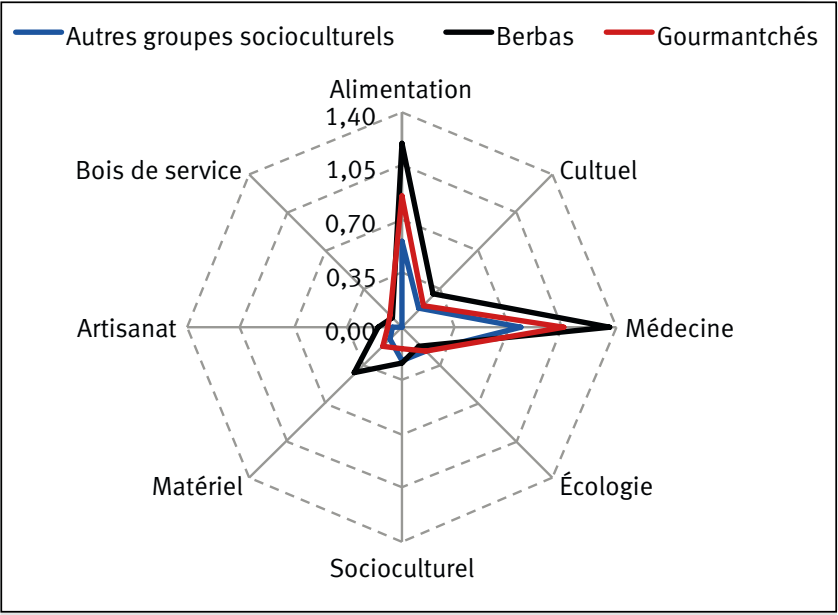

Figure 4.

Diagramme en radar décrivant l'importance culturelle de Bombax costatum en fonction des groupes socioculturels. socioculturels » tandis que la graine est plus importante pour les Gourmantchés. Contrairement aux Gourmantchés et aux « autres groupes socioculturels » qui ont plusieurs préférences (calice, écorce et jeune tige), les Berbas préfèrent seulement les calices (figure 5 ).
Tableau IX.

Variation de l'importance culturelle de Bombax costatum en fonction de ses organes/parties de plante : résultats du test de Kruskal-Wallis.

\begin{tabular}{|c|c|c|c|}
\hline $\begin{array}{l}\text { Organe ou } \\
\text { partie de plante }\end{array}$ & ddl & Statistique H & Prob. \\
\hline Calice & 2 & 0,74 & $0,691^{\text {ns }}$ \\
\hline Écorce & 2 & 9,87 & $0,007^{\star *}$ \\
\hline Feuille & 2 & 12,64 & $0,002^{* *}$ \\
\hline Fleur & 2 & 3,48 & $0,175^{\text {ns }}$ \\
\hline Graine & 2 & 9,17 & $0,010^{*}$ \\
\hline Jeune tige & 2 & 6,54 & $0,038^{*}$ \\
\hline Racine & 2 & 0,16 & $0,921^{\text {ns }}$ \\
\hline \multicolumn{4}{|c|}{$\begin{array}{l}\text { Codes du niveau de significativité : } \\
0={ }^{\star \star \star} ; 0,001={ }^{\star \star} ; 0,01={ }^{*} ;>0,05=\text { ns. } \\
\mathrm{H} \text { : statistique de Kruskal-Wallis ; ddl : degré de liberté } \\
\text { Prob. : probabilité liée au test statistique. }\end{array}$} \\
\hline
\end{tabular}




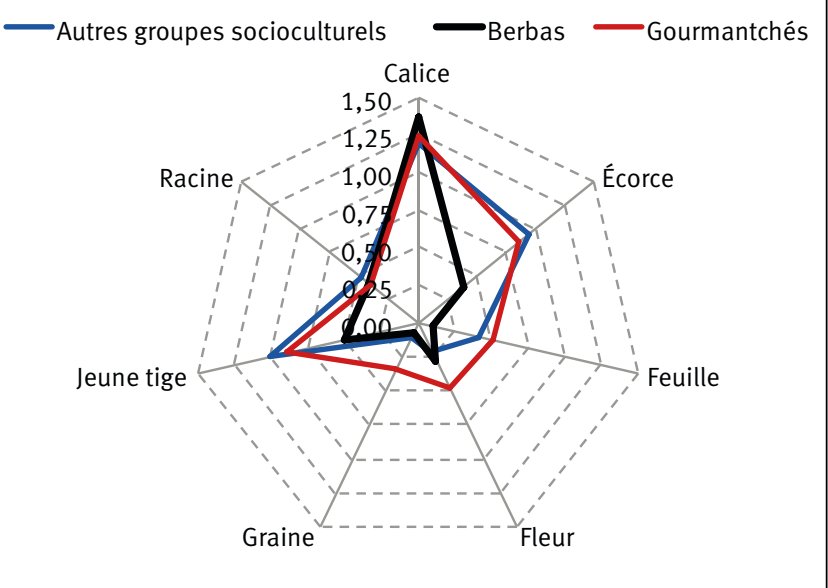

Figure 5.

Diagramme en radar décrivant l'importance culturelle des organes de Bombax costatum en fonction des groupes socioculturels.

\section{Discussion}

Cette étude sur $B$. costatum, la première du genre au Bénin, est une contribution à la meilleure connaissance des PFNL et de leur potentiel pour l'amélioration des conditions de vie des populations rurales. De nombreuses études se sont focalisées, au cours de cette dernière décennie au Bénin, sur l'importance culturelle des plantes endogènes (Dadjo et al., 2012 ; Fandohan et al., 2010 ; Ekué et al., 2010 ; Koura et al., 2011 ; Gouwakinnou et al., 2011). Toutefois, aucune d'entre elles n'a décrit les usages de $B$. costatum.

Chaque groupe socioculturel désigne $B$. costatum par une appellation spécifique, révélant un contact et une tradition lointains de l'espèce dans l'histoire des peuples vivant autour de la réserve de biosphère de la Pendjari. Les Gourmantchés et les Natimbas l'appellent « arbre léger » à cause probablement de leur utilisation de son bois, par rapport à d'autres groupes socioculturels. Ils l'utilisent plus dans la catégorie d'usages "matériel » et constatent qu'il est facile à couper. Toutefois, ceci peut constituer une menace pour la conservation de l'espèce en milieu Gourmantché et Natimba. Quant aux Peuhls, Berbas et Waamas, ils préfèrent l'appeler " arbre à fleur rouge ", en lien avec la couleur rouge de ses fleurs qui apparaissent en saison sèche et sont très remarquables dans le paysage. De pareilles différences dans les appellations par groupe socioculturel ont été notées par Gruca et al., (2015) pour Borassus aethiopum au Ghana. Dans le cas présent, il est plausible que l'appellation ait un lien avec l'usage préférentiel de l'espèce pour chaque groupe socio-linguistique.

$\mathrm{Au}$ total, l'étude a permis de recenser 46 usages de l'espèce, classés en huit catégories d'usages, à savoir : alimentation, artisanat, bois de service, cultuel, écologique, matériel, médecine et religion. Les catégories d'usages les plus importantes sont l'alimentation, la médecine, le cultuel et celle des avantages écologiques. Ces résultats montrent une grande diversité des usages de l'espèce dans les villages riverains de la Pendjari, contrairement aux résultats de Belem (2009) au Burkina Faso, pays voisin où l'espèce est plus connue pour être utilisée essentiellement en alimentation humaine et en artisanat. Cette variation non négligeable des connaissances des propriétés de l'espèce pourrait s'expliquer par les différences culturelles entre groupes socioculturels et les besoins spécifiques à chaque peuple. Au-delà des considérations culturelles, ce résultat pourrait également être expliqué par le fait que les usages additionnels de l'espèce au Bénin sont assurés par d'autres espèces plus distribuées et abondantes au Burkina Faso, tel que stipulé par l'hypothèse de saisonnalité (Albuquerque, 2006). Cette hypothèse prédit en effet que les ressources préférées et plus utilisées sont les ressources locales les plus disponibles. Toutefois, cette relation peut être modifiée par des considérations économiques et sociales (Albuquerque, 2006). En effet, la différence observée au plan médicinal pourrait aussi s'expliquer par la préférence des populations riveraines pour les plantes médicinales endogènes, parfois allant vers une contrainte, n'ayant plus d'autres alternatives de traitement proches (Albuquerque, 2006).

Le calice est l'organe le plus utilisé, généralement sous forme de sauce légume ou sauce gluante. Les usages alimentaires et médicinaux, en particulier à partir des fruits ou fleurs ou encore des calices, ont souvent été rapportés comme les plus prépondérants au sein de plusieurs communautés (Assogbadjo et al., 2008 ; Gouwakinnou et al., 2011). Ces résultats peuvent s'expliquer par le fait que les populations locales ont pour premier objectif la satisfaction des besoins vitaux, dont les besoins alimentaires (Shackleton et Shackleton, 2004), ce qui les amène à exploiter considérablement les parties comestibles des espèces. Cette forte valorisation de l'espèce pour les usages alimentaires constitue un atout pour la sécurité alimentaire dans ces zones d'occurrence. $B$. costatum est également fortement sollicité (87\%) dans la médecine traditionnelle pour la composition des remèdes à certaines maladies : paludisme, fièvre, dermatoses, fortifiant, colique, convulsion, pansement de plaies, antidouleur, inflammation des pieds, hémorroïdes, abcès, régulation de la température, facilitation à l'accouchement, mitose, œdème, hernie et épilepsie. Cette diversité des usages médicinaux de la plante constitue également un atout pour des explorations futures de plusieurs propriétés médicinales à travers des études ethno-pharmacologiques.

$B$. costatum est à la fois utile aux hommes et aux animaux. En effet, les fleurs de $B$. costatum sont mellifères, de plus, elles attirent également les oiseaux. Ces résultats s'accordent avec ceux de Belem et al. (2009) qui ont observé que les abeilles se nourrissent du nectar des fleurs de l'espèce.

Les résultats ont par ailleurs montré que la connaissance sur les usages et de l'importance culturelle de l'espèce varie suivant les groupes socioculturels. Les Gourmantchés et les Berbas sont les groupes socioculturels qui utilisent le plus l'espèce, et qui lui accordent une grande importance culturelle. Nous suggérons l'intégration des communautés étudiées et de leurs savoirs sur l'espèce dans les stratégies d'utilisation durable de l'espèce en organisant la chaîne de commercialisation de la collecte des calices, en introduisant la culture de l'espèce dans les jardins de case au sein des terroirs villageois autour de la réserve de biosphère de la Pendjari, enfin, en substituant d'autres espèces à croissance rapide à ses usages comme matériaux de construction. 


\section{Conclusion}

Cette étude ethnobotanique de Bombax costatum au sein des groupes socioculturels vivant autour de la réserve de biosphère de la Pendjari confirme l'importance de l'intégration de la culture de l'espèce dans les systèmes agroforestiers pour une diversification des filières agricoles et la conservation de la biodiversité. Bien que l'espèce soit utilisée par tous les groupes socioculturels, elle demeure sous-valorisée sur le marché local et international malgré ses atouts alimentaires et médicinaux. L'importance de l'usage alimentaire des calices indique un potentiel de l'espèce dans les stratégies d'amélioration de l'état nutritionnel basé sur les ressources locales. De même, le fort potentiel de l'espèce en médecine traditionnelle suggère des études pharmacologiques en vue d'une probable identification et extraction de ses principes actifs. Ceci pourrait être une source de revenus pour les ménages ruraux. Nous suggérons que cette étude soit étendue à d'autres localités d'occurrence de l'espèce et au sein d'autres groupes socioculturels, afin d'identifier d'autres utilisations possibles de l'espèce pour une meilleure valorisation de ses potentialités. L'évaluation de la valeur nutritionnelle des calices, des revenus générés par sa commercialisation et de l'identification des probables morphotypes est également capitale pour la valorisation de la plante. Les études de propagation entamées par Belem (2009) devront également être poursuivies en vue de la maîtrise de la culture de l'espèce.

\section{Références bibliographiques}

Adomou C. A., 2005. Vegetation patterns and environmental gradients in Benin. Implications for biogeography and conservation. PhD thesis, Wageningen University, Wageningen, The Netherlands, $136 \mathrm{p}$.

Akaike H., 1973. Information theory as an extension of the maximum likelihood principle. In: Petrov B. N., Csaki F. (eds). Second International Symposium on Information Theory. Budapest, Hungary, Akademiai Kiado, 267-281.

Albuquerque U. P., 2006. Re-examining hypotheses concerning the use and knowledge of medicinal plants: A study in the Caatinga vegetation of NE Brazil. Journal of Ethnobiology and Ethnomedicine, 2: 30.

Arbonnier M., 2002. Arbres, arbustes et lianes des zones sèches d'Afrique de l'Ouest. Deuxième édition. Cirad, MNHN, 574 p.
Assogbadjo A. E., Glèlè Kakaï R., Chadaré F. J., Thomson L., Kyndt T., Sinsin B., Van Damme P., 2008. Folk classification, perception, and preferences of baobab products in West Africa: Consequences for species conservation and improvement. Economic Botany, 62: 74-84.

Belem B., Smith O. C., Theilade I., Bellefontaine R., Guinko S., Lykke A.M., et al., 2008. Identification des arbres hors forêt préférés des populations du Sanmatenga (Burkina Faso). Bois et Forêts des Tropiques, 298 (4) : 53-64. http:// bft.cirad.fr/cd/BFT 298 53-60.pdf

Belem B., 2009. Ethnobotanique et conservation de Bombax costatum Pel. \& Vuil. (faux kapokier) dans les systèmes de production agricoles du plateau central, Burkina Faso. Thèse de doctorat, Université de Ouagadougou, Burkina Faso, 189 p.

Camou-Guerrero A., Reyes-Garcia., Martinez-Ramos M., Cassa A., 2008. Knowledge and use value of plants species in a Raramuri community: a gender perspective for conservation. Human Ecology, 36: 259-272.

Cavendish W., 2000. Empirical regularities in the poverty environment relationship of rural households: evidence from Zimbabwe. World Development, 28 (11): 1979-2003.

CENAGREF, 2009. Plan d'aménagement participatif et de gestion (2004-2013). Cotonou, Bénin, Cenagref, 124 p.

Codjia J. T. C., Assogbadjo A. E., Mensah M. R., 2003. Diversité et valorisation au niveau local des ressources forestières alimentaires du Bénin. Cahiers Agricultures, 12 : 321331. http://revues.cirad.fr/index.php/cahiers-agricultures/ article/view/30405

Dagnelie P., 1998. Statistique théorique et appliquée. Tome 1. Bruxelles, Belgique, De Boeck et Larcier, 517 p.

Dadjo C., Assogbadjo A. E., Fandohan B., Glèlè Kakaï R. L., Chakeredza S., Houéhanou T. D., Sinsin B., 2012. Uses and management of black plum (Vitex doniana Sweet) in Southern Benin. Fruits, 67 (4): 239-248. https://doi.org/10.1051/ fruits/2012017

Dytham C., 2011. Choosing and using statistics: a biologist's guide. John Wiley \& Sons, 287 p.

Ekué M. R. M., Sinsin B., Eyog-Matig O., Finkeldey R., 2010. Uses, traditional management, perception of variation and preferences in ackee (Blighia sapida K.D. Koenig) fruit traits in Benin: implications for domestication and conservation. Journal of Ethnobiology and Ethnomedicine, 6: 12.

Fandohan A. B., Gouwakinnou N. G., Déléké Koko K. I. E., Glèlè Kakaï R., Assogbadjo A. E., 2015. Domesticating and conserving indigenous trees species: an ecosystem based approach for adaptation to climate change in Sub-Sahara Africa. CAMES (Conseil Africain et Malgache pour l'Enseignement Supérieur), Sciences de la vie, de la terre et agronomie, 3 (1): 55-60.

Fandohan B., Assogbadjo A. E., Glèlè Kakaï R., Kyndt T., De Caluwe E., Codjia J. T. C., Sinsin B., 2010. Women's traditional knowledge, use value and the contribution of tamarind (Tamarindus indica L.) to rural households' cash income in Benin. Economic Botany, 64 (3): 248-259. 
FAO, 2001. Produits forestiers non ligneux 13. Évaluation des ressources en produits forestiers non ligneux. Rome, Italie, FAO, $139 \mathrm{p}$.

Gomez-Beloz A., 2002. Plant use knowledge of the Winikina Warao: The case for questionnaires in ethnobotany. Economic Botany, 56: 231-241.

Gouwakinnou G. N., Lykke A. M., Assogbadjo A. E., Sinsin B., 2011. Local knowledge, pattern and diversity of use of Sclerocarya birrea. Journal of Ethnobiology and Ethnomedicine, 7: 8 .

Gruca M., Blach-Overgaard A., Balslev H., 2015. African palm ethno-medicine. Journal of Ethnopharmacology, 165: 227-237.

Honfo H., Tovissodé F. C., Gnanglè C., Mensah S., Salako V. K., Assogbadjo A. E., et al., 2015. Traditional knowledge and use value of bamboo in Southeastern Benin: implications for sustainable management. Ethnobotany Research and Applications, 14: 139-153.

Houehanou T. D., Assogbadjo A. E., Kakaï R. G., Houinato M., Sinsin B., 2011. Valuation of local preferred uses and traditional ecological knowledge in relation to three multipurpose tree species in Benin (West Africa). Forest Policy and Economics, 13 (7): 554-562.

Ingram V., Bongers G., 2009. Valuation of Non-Timber Forest Product Chains in the Congo Basin: A methodology for valuation. CIFOR, Younde, Cameroon, FAO-CIFOR-SNV-World Agroforestery Center-COMIFAC, $80 \mathrm{p}$.

Johnson J. B., Omland K. S., 2004. Model selection in ecology and evolution. Trends in Ecology and Evolution, 19 (2): 101-108.

Koura K., Ganglo J. C., Assogbadjo A. E., Agbangla C., 2011. Ethnic differences in use values and use patterns of Parkia biglobosa in Northern Benin. Journal of Ethnobiology and Ethnomedicine, 7 (1): 42.

Lescuyer G., 2010. Importance économique des produits forestiers non ligneux dans quelques villages du Sud-Cameroun. Bois et Forêts des Tropiques, 304 (2) : 15-24. http:// bft.cirad.fr/cd/BFT 304 15-24.pdf

Mahapatra A. K., Albers H. J., Robinson E. J. Z., 2005. The Impact of NTFP sales on rural households' cash income in India's dry deciduous forest. Environmental Management, 35 (3): 258-265.

Ouédraogo I., Nacoulma B. M. I., Ouédraogo O., Hahn K., Thiombiano A., 2014. Productivité et valeur économique des calices de Bombax costatum Pellegr. \& Vuillet en zone soudanienne du Burkina Faso. Bois et Forêts des Tropiques, 319 : 31-41. http://bft.cirad.fr/cd/BFT 319 31-41.pdf
Oyen L. P. A., 2011. Bombax costatum Pellegr. \& Vuillet. [Internet] Fiche de PROTA4U. Brink M., Achigan-Dako E. G. (Éditeurs). PROTA (Plant Resources of Tropical Africa / Ressources végétales de l'Afrique tropicale), Wageningen, PaysBas. http://www.prota4u.org/search.asp

R Core Team, 2016. R: A language and environment for statistical computing. Vienna, Austria, R Foundation for Statistical Computing. https://www.R-project.org/

RGPH4 ( $4^{\mathrm{e}}$ Recensement général de la population et de l'habitation), 2015. Rapport provisoire de l'Institut national de la statistique et de l'analyse économique. Cotonou, Bénin, 33 p.

Shackleton C., Shackleton S., 2004. The importance of non-timber forest products in rural livelihood security and as safety nets: a review of evidence from South Africa. South African Journal of Science, 100 (11-12): 658-664.

Sofowara A., 2010. Plantes médicinales et médicine traditionnelle d'Afrique. Paris, France, Karthala, 378 p.

Tardio J., Pardo-De-Santayana M., 2008. Cultural importance indices: a comparative analysis based on the useful wild plants of southern Cantabria (northern Spain). Economic Botany, 62 (1): 24-39.

Vodouhê G. F., Coulibaly O., Greene C., Sinsin B., 2009. Estimating local values of non-timber forest products to Pendjari Biosphere Reserve dwellers in Benin. Economic Botany, 63 (4): 397-412. 